\title{
Multi-Agent Decision Making Architecture and Distributed Control
}

\author{
Vladimír Mařík, Karel Kraus, Ondřej Flek, Jan Bezdiček \\ E-mail: \{marik, karel, flek, jan\}@abrcp.cz \\ Czech Technical University \\ Rockwell Automation Ltd. \\ Faculty of Electrical Engineering \\ Technická 2 \\ Research Center Prague \\ 16627 Prague 6 \\ Americká 22 \\ Czech Republic \\ 12000 Prague 2 \\ Czech Republic
}

\begin{abstract}
The paper presents principles behind a multi-agent system DISCIM supporting distributed decision making. Using analogical ideas, a distributed architecture for control engineering purposes has been proposed. Each node (agent) in this architecture is expected to consist of two parts: a stand-alone control device and a wrapper. Each part runs on an independent hardware (processor). Standardized communication interfaces/languages are considered. The solution of a reconfigurability problem is described in detail. Brief comparison of the multi-agent systems for decision making and distributed control is presented.
\end{abstract}

\section{Keywords}

Multi-Agent Systems, Distributed Control, System Reconfiguration

\section{INTRODUCTION}

The growing complexity of problems to be solved in industry requires new overall software system architectures integrating the already existing "islands" of well-working algorithms into more robust global systems. This is true for both the expert/decision making systems and for the complex control systems.

In the area of decision making systems, this integration trend has resulted in various blackboard architectures designed for distributed knowledge processing or for integration of distributed knowledge bases. Such approach is based on the ideas of Distributed AI. The results 
seem to be too cumbersome and not general enough in the case the global system should be quite open and aimed at exploration of knowledge sources of different nature. The multi-agent technology (Wooldridge, 1995) connected with the OO-style of programming is currently considered to be much more suitable for creating open, flexible environment able to integrate software pieces of diverse nature written in different languages and running on different types of computers.

The multi-agent approach is currently being used in many subareas of CIM, e.g. in scheduling (Rabelo, 1995), in concurrent engineering (Osorio, 1995) or for database integration purposes (Afsarmanesh, 1994). In the area of control systems, various distributed control architectures were presented by the universities as well as by different vendors. These are either based on existence of some central coordinating unit or try to explore (more or less) the ideas behind multi-agent systems.

Although the way of thinking of engineers is rather object-oriented than procedural in its nature, the multi-agent systems based usually on object-oriented programming technology "penetrate" into practical applications only rarely, rather exceptionally. The engineering community of industrial engineers is currently not well prepared to accept the new vision. The acceptance of this multi-agent vision requires a deep understanding of the object-oriented philosophy and substantially new way of thinking.

The majority of industrial control engineers prefer "classical", procedural, sequential programming. Our experience says that - even using the distributed control tools and technologies - they usually try to reduce the multi-agent ideas behind the distributed control. Their tendency is to have a detailed overview of the activity of each control element under their strict global control. Many engineers in industry do see the reduced number of wires as the main advantage of the distributed control. Unfortunately, they underestimate the real main advantages of the multi-agent control: high modularity, simple and easy reconfigurability/extendibility of the control architecture and software reusability.

These advantages of the multi-agent architectures are quite clearly visible and much better understandable in the case of distributed decision making systems. As a matter of fact, this area can be considered as a pioneering application field of multi-agent ideas. One reason for it is its close connection to the AI. We propose to use the experience gathered during the design, development and implementation of multi-agent decision making systems/tools in designing efficient architecture of distributed control systems.

\section{DISCIM ENVIRONMENT}

Our goal was to design, develop and implement a comparatively open multi-agent environment suitable for efficient creating of complex expert or decision support systems. It is expected that this environment is able to integrate geographically distributed knowledge sources or problem solving units.

The task under consideration is located just on the borderline between Software Engineering and Artificial Intelligence. The idea of software integration based on efficient communication among parallel computational processes as well as that of the open architecture (enabling to add new elements without any change in the others) have been provided by the Software Engineering area. On the other hand, the multi-agent approach stemming from the theory of agency, from behavioral models of agents and methods of agentification of standalone programs can be considered as a contribution of Artificial Intelligence. 
The name of the developed environment DISCIM reflects its first intended application building a geographically DIStributed decision making system for CIM purposes within the EU-PECO project EUROSAT No. 9645 "CIM via Satellite" (Mařík, 1994).

The following principles have been used to design the DISCIM system:

a) The agents are independent, autonomous entities communicating in a peer-to-peer way among themselves. The asynchronous message passing/broadcasting in UNIX/INTERNET environment is used to perform this communication.

b) Each agent consists of a functional body (usually a stand-alone program with a welldefined functionality) and a wrapper (which is responsible for involvement of the agent into the community of agents). The wrapper contains a model of the agent's behavior in the form of the list of classes in the Eiffel notation. Some classes represent messages which can be received and sent out by the agent. As a matter of fact, the wrapper translates the interagent communication into the instructions for the activity of the functional body and mediates the results of the body activity back to the agents' community.

c) The typical model of an agent's behavior can be described in the following way: At the very beginning, the agent is initialized (included into the agents' community). Then it observes the messages broadcasted within the community. In the case, the knowledge contained in the agent is required by some member of the agents' community, the agent sends out a confirmation message (informs the requesting agent that it is ready to solve the given sub-problem) and initializes its own body. If the body requires some piece of information from the others in the community, the wrapper broadcasts this bid. The agent then waits (for a pre-set time period) for any confirmation message from some of the agents; the agent which confirms the ability to provide the required piece of information as the first one is considered (the required information may be possessed by more than one agent). If no confirmation message has occurred within the pre-set time interval, the original agent replaces the required piece of data by a default value.

The model of behavior contained in the wrapper describes only the re-active part of behavior (in the sense of Wooldridge's and Jenning's classification (Wooldridge, 1995)). The deliberative behaviour which relies on explicit, internally held symbolic models (if such behaviour is required) is expected to be implemented as a natural part of the agent's body. Of course, the re-active behavioral model can be enriched by extending the list of classes or messages to be received and sent out.

d) There is no central memory or control in the agents' community. Similarly to ARCHON (Jennings, 1993), the corresponding pieces of the control strategy are "owned" by the individual agents or meta-agents, respectively, they are embodied into the individual behavioral models as rules contained in the classes (input/output messages description).

e) There is a library of standard classes/messages (written in the object-oriented programming language Eiffel) available for the environment user (= application designer). This library, called "Eiffel PVM Library" helps him/her to create his/her own agents by very fast agentification of arbitrary stand-alone programs.

f) User interface has been designed as a separate agent in the community. It is not only an agent responsible for communication with the user. It is the only agent which can initialize the activity of the agents' community. 
g) The basic types of agents currently developed and tested are:

- an expert system agent

- a database agent (as a body of which the POSTGRES OO-database is considered and POSTGRES' own standard query language is considered),

- a qualitative simulation agent (exploring the QUASIMODO (Štěpánková, 1994) system for qualitative simulation, written in $\mathrm{ECL}^{i} \mathrm{PS}^{e}$ version of Prolog $\left(\mathrm{ECL}^{i} \mathrm{PS}^{e}\right.$, 1992)) and

- a user interface agent.

- a neural network agent running on a set of 148 transputers.

h) A meta-agent (under development) is aimed at permanent observing of the multi-agent community and its reconfiguration if necessary. The main goal of the meta-agent is

- to discover a failure of a particular agent (caused e.g. by loosing the Internet connection or by fatal failure of the corresponding computer),

- to re-distribute the load (by either creating a new agent elsewhere, or by changing some functionalities of the other agents etc.). The activity of this agent is based on the "cluster and clone" technique (Maturana, 1995). For each specific functionality there is a (more or less) virtual cluster (subset) of agents. The reconfiguration or load relocation problem should be solved within the given cluster. This problem is usually easily solved by creating a new process (agent) which is a copy ("clone") of some cluster member. Typically, to one cluster of agents one corresponding meta-agent is assigned to manage the reconfiguration/relocation problem at hand.

i) The crucial problem of the environment implementation was the problem how to ensure the inter-agent peer-to-peer communication in the UNIX/INTERNET environment (Mařik, 1994). Finally, it was decided to explore the advantages of the PVM (Parallel Virtual Machine) system. PVM (PVM, 1993) is a software package that enables concurrent computing on loosely coupled network of processing elements. PVM may be implemented on a hardware base consisting of different machine architectures interconnected by one or more networks of different types.

\section{MULTI-AGENT CONTROL ARCHITECTURE}

The aim of the rest of this paper is to outline some possibilities of the future development of distributed control architecture exploring the ideas behind the DISCIM system.

The original idea of the "Distributed Control System" was the idea of "smart" devices connected to the communication network using very cheap components (low-end processors, network controllers, etc.). It was expected that this solution should enable direct connection of some rather simple/cheap devices (i.e., pushbuttons, pilot lights, limit switches) directly to the network and their peer-to-peer communication. The main benefit of such a solution was expected to be lower wiring and installation cost. However, the experience with real prototypes/products of this type shows that it is very hard to implement other (and in fact much more important) features of the distributed control systems like high flexibility, easy programming, simple reconfiguration, high reusability of both the hardware and software, possibility to use devices of different classes (e.g., powerful PLCs and simple pushbuttons) as an organic part of one distributed control system, etc. There are usually two main bottlenecks - 
the lack of computing power in the devices and the complexity of the programming environment which has to be general enough to fulfill two contradictory demands (namely to provide the user with the tools for programming the whole distributed control application as well as allow to reuse the already existing pieces of code created for example by the OEMs (Original Equipment Manufacturers) easily.

The multi-agent approach described in this paper is capable to overcome the above mentioned weaknesses of the "cheap" low-end solution easily. Certainly this approach has its own disadvantages, too. There are two main problem areas which have to be taken into account:

1. Relatively high price of the hardware,

2. Very complex programming environment.

Fortunately, neither of these two obstacles is critical. Analyzing the problem the following conclusions could be reached:

\section{Price of the components}

The higher price of the components is paid off by much higher flexibility of control solutions, by software reusability, and by winning the competitive advantage.

\section{Complex programming environment}

It is probably unrealistic to create a general purpose programming environment for distributed control capable to handle all the devices (including those that will be designed in the future). On the other hand it is fairly easy to use an empty shell (off-the-shelf product) capable to integrate various programming packages for different devices. A software package like fm/Aspects (Holas, 1995) can be a typical example of such a high-level programming environment. The main advantage of this solution is that it allows a simple encapsulation of the code related to a certain logical subpart of the whole distributed control system.

Although the following text is intended to describe the new architecture mainly on theoretical level, it is designed in such a way that it is possible to implement the ideas using the technology that is already available.

Currently, various control networks are being used (CAN, DeviceNet, Interbus-S, Profibus, Ethernet, etc.) in industrial practice to interconnect various equipment involved into an automated production system. Each unit to be connected to any of these networks must be equipped with appropriate hardware and software serving the communication between the unit and the network. Usually each of the networks uses different hardware and different network protocols, so the unit has to have a different interface into each of the networks. Typically, there is an extra version of each type of the device suitable for connecting to each of the networks. Thus, connecting a device to a new type of network requires to design a new type of device adapted to the new network.

It seems to be advantageous to introduce a more modular and open architecture making it easier to connect any of the devices to any required network. To achieve this goal a multi-agent approach, concept used and verified in distributed artificial intelligence, could be explored. Any device connected to the network would be regarded as an autonomous agent. Such an agent consists of the device itself, enwrapped into a wrapper serving the communication and interconnection purposes. The wrapper is an element containing interfaces to both the external world and to the device, mediating the information interchange between the device and the network. This solution has several advantages: 
- The wrapper can be universal, e.g. there could be a wrapper specialized to a particular type of network, but all the devices to be connected to one type of network could use the same type of wrapper.

- Using a standard Language $X$ for communication between the device (body) and the communication module (wrapper) assumes, that all the devices designed for use with a distributed control system have a standard Language $X$ interface. This would force designers to enable the devices to communicate with others in a more standardized manner.

- It is possible to use clearly an object-oriented approach to "programming" of such a system due to the possibility to split the problem oriented part of the unit (i.e., the device itself) and the communication module (the wrapper). Thus, the codes of both parts could be independent, enabling a better readability, transparency and adaptability of the software. As the problem-oriented and communication tasks are separate, they do not interfere with each other.

- If the need for connecting the device to another network type occurs, only a new wrapper would have to be designed (very often the new wrapper is a modification of some already existing one), taking into account properties of the new network type, but the interface to the device could remain the same. If, for example, a network with much higher communication speed would be used, the code serving the communication would have to be changed. In a classical approach, this change would require rewriting not only of the communication part of the code, but of the problem oriented part of the code as well. If the two parts are separate, then only the communication part of the code needs to be modified.

To implement such a system the following issues should be studied:

- Designing a network protocol suitable for use with more possible network types would provide a background for designing even more general wrapper, i.e., the differences between wrapper modules for various kinds of network would be less extensive.

- An interface between the wrapper module and the device should be designed. Some kind of shared memory seems to be a suitable medium for communication. In this case, the communication between both parts of the unit would be carried out using an data table structure. A semaphore mechanism would have to be used to control the communication.

- As the information to be interchanged varies from device to device, it is necessary to make the protocol used for the communication between both parts of the unit general enough to match all the possible device types.

\section{SYSTEM DESCRIPTION}

\subsection{Network}

A typical distributed control system is shown in Figure 1. The basis of the system is a network (enabling the communication among the nodes), which can be chosen from a wide range of networks available, e.g. CAN or Ethernet. A number of distributed control nodes is connected to the network. The structure of the nodes will be described later. Various nodes can represent e.g. drives, limit switches, proximity switches, operator terminals, PLCs, push button panels, etc. Except of these nodes, one or more nodes are computers (PCs) serving - similarly to DISCIM - as meta-agents. The task of these meta-agents is to organize work of a vir- 
tual cluster of other nodes. The cluster of nodes is usually a group of nodes having approximately the same functionality or being connected to one physical system. The virtual clusters can overlap each other. The meta-agent contains a network configuration information, it may store programs of some of the devices, and may solve autoconfiguration tasks as well.

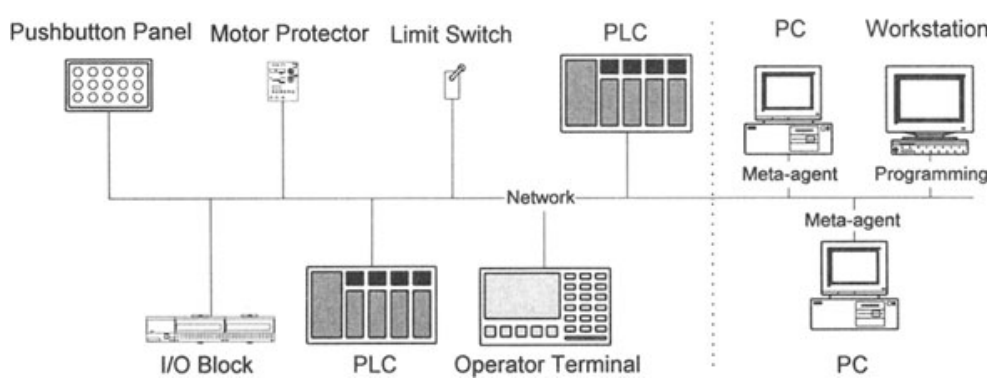

Figure 1.

A typical distributed control system is shown in Figure 1. The basis of the system is a network (enabling the communication among the nodes), which can be chosen from a wide range of networks available, e.g. CAN or Ethernet. A number of distributed control nodes is connected to the network. The structure of the nodes will be described later. Various nodes can represent e.g. drives, limit switches, proximity switches, operator terminals, PLCs, push button panels, etc. Except of these nodes, one or more nodes are computers (PCs) serving - similarly to DISCIM - as meta-agents. The task of these meta-agents is to organize work of a virtual cluster of other nodes. The cluster of nodes is usually a group of nodes having approximately the same functionality or being connected to one physical system. The virtual clusters can overlap each other. The meta-agent contains a network configuration information, it may store programs of some of the devices, and may solve autoconfiguration tasks as well.

The nodes (agents) communicate with each other by sending messages. These messages may be either broadcast messages (intended for all the nodes in the system), or they can be sent point-to-point. The messages can obtain two basic types of information:

- Runtime information (input/output data, communication between two PLCs, etc.),

- Configuration information (configuration data, programs).

The system may be programmed* from a specialized computer (let's say a workstation, or a PC) which is considered to be a specific agent. The program is then transported into the meta-agent node or other agents. The particular parts of the program are downloaded into the particular nodes when necessary. After programming of the system is finished, the programming workstation can be disconnected from the network.

\footnotetext{
* Programming of the system consists of downloading up to 3 sets of data (configuration, program, communication configuration data - see Figure 2 ) to each of the nodes.
} 


\subsection{Node}

From the point of view of the multi-agent theory, the node acts as an agent of a multi-agent system. It is a unit consisting of the device (the body) and the communication module (the wrapper), together creating one subject in the network. Both basic parts of the node (agent) are described in the following text and shown in Figure 2.

\subsubsection{Device (Body)}

Device is a piece of hardware and software (e.g. drive, PLC, limit switch, operator terminal) which is to be connected into the distributed control system.

Firmware/Operating System: Either a device firmware (e.g. in a case of a motor protector), or an operating system (e.g. if the device is a PLC).

Configuration: This configuration can be provided in both the hardware (switches) or the software (downloadable configuration table) form. In case of the software configuration it may be downloaded directly into the device using a hand-held terminal, or it may be provided by the meta-agent or by the programming workstation through the network.

Program: A program performing the function of the device. Just like in the case of software configuration, the device can be programmed both locally or from the meta-agent computer. This program can be very simple (in a simple device) or can be extremely complex (e.g. in a case of a PLC).

Shared Memory: This piece of hardware mediates all the communication between the device and the communication module of the node. This memory may be read and written to by the means of the Language $X$. This memory would probably be resident in the device module of the node. It would enable to change its capacity according to the demand of various types of device (a PLC would probably communicate more data than a motor protector, so it would require a memory of bigger capacity to be able to transfer all the necessary information).

Language $X$ : This language serves to the information interchange between the device (body) and the communication module (wrapper) parts of the node. If either one or the parts is to provide some information to the other part, it must use functions of the Language $X$. There should be two possible approaches to inter-module communication. Language $X$ should provide means enabling both ways of communication:

- event driven - if an event requiring to transfer some data occurs, the sending device sends a "change-of-state" signal to the other module and then transfers the data using Language $X$ services,

- polling - the device writes all the data into a predefined part of the shared memory. The communication module regularly scans that particular memory area (using the Language $X$ services) and waits for some new data to occur. 


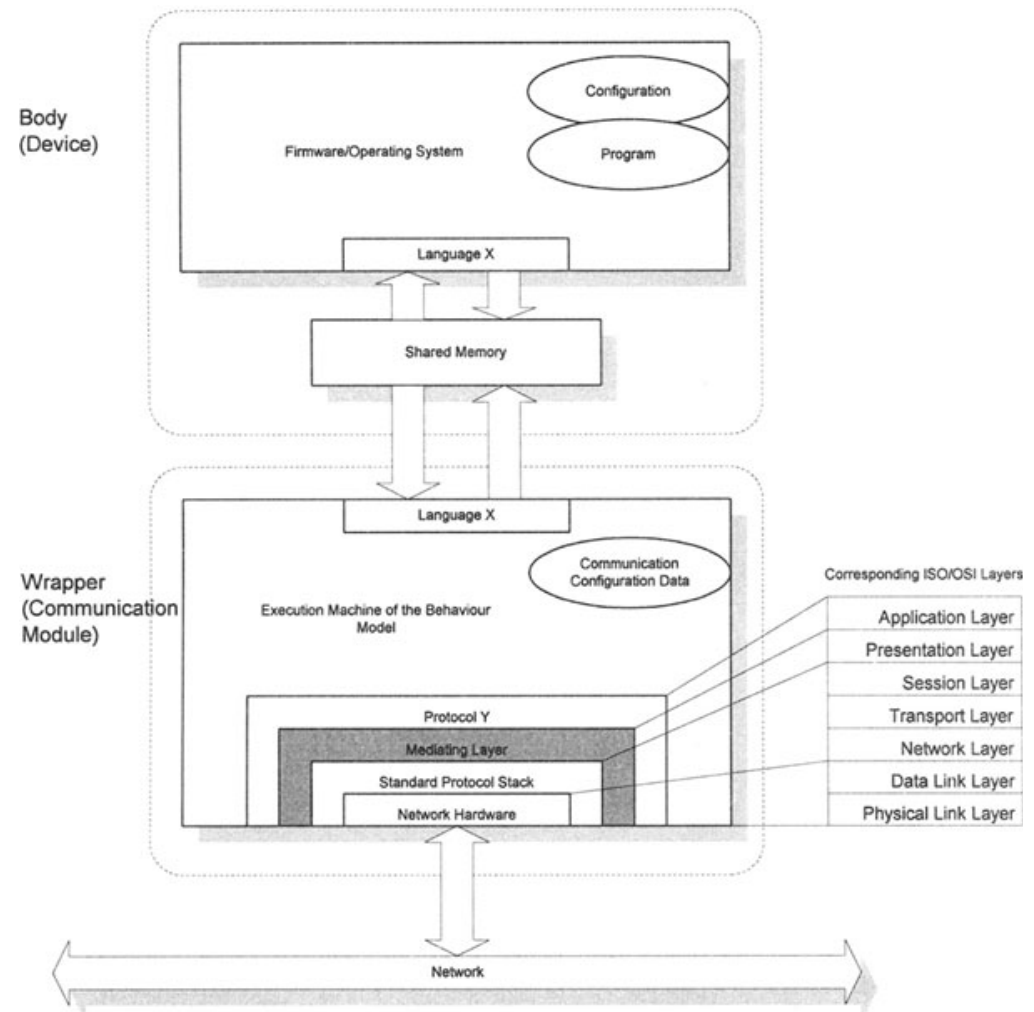

Figure 2.

\subsubsection{Communication Module (Wrapper)}

This module serves to connecting the device into the distributed control system. This module should be universal to all the devices to be connected to the system, or, at least, to a particular group of devices of a similar type.

Internal Model Execution Machine: The operation of this part can be described by a finite automaton. One of the possible descriptions is shown in Figure 3. Using the dynamic data (described bellow) as its "program", it accomplishes the model of the agent's behaviour. It controls the overall communication strategy of the node in the system. It defines the actions of the node performed to connect to the meta-agent, to download the configuration and/or program and then controls the basic requirement-handling cycle. This cycle is general and is the same no matter what the device is. 


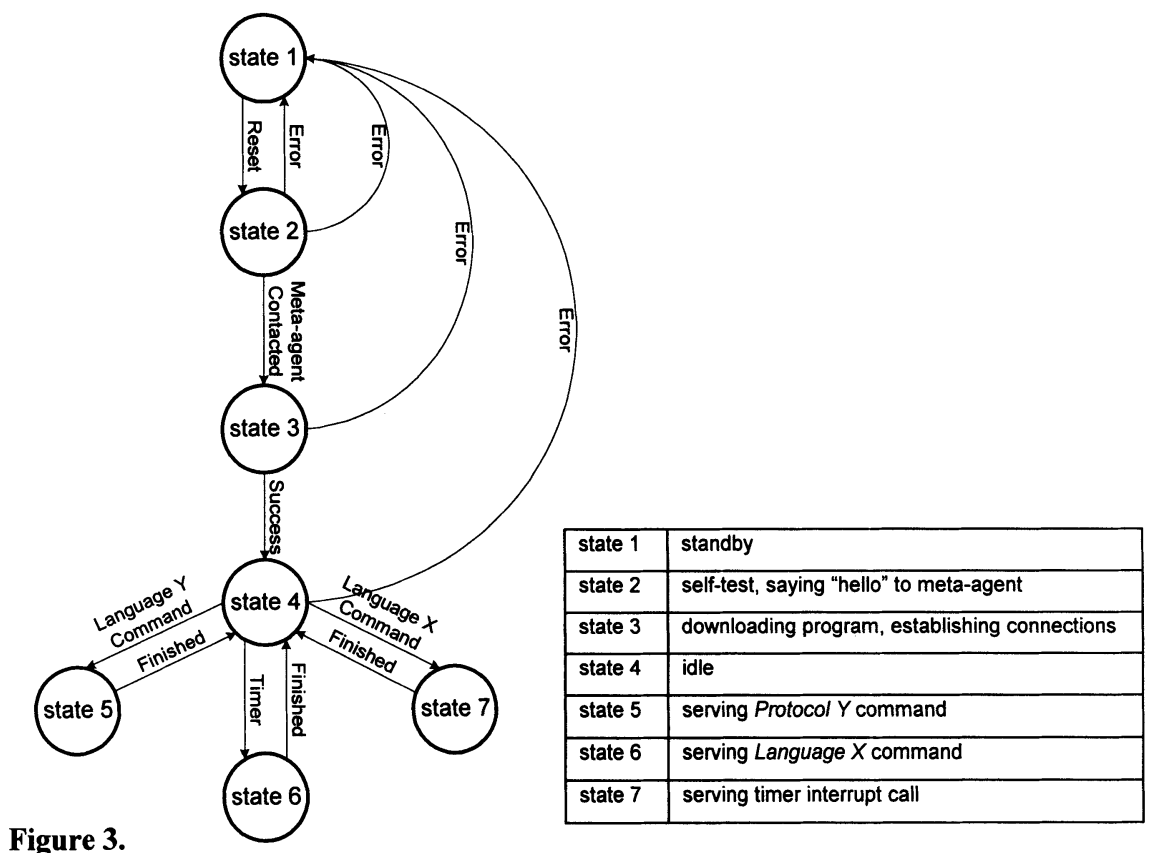

Figure 3.

Dynamic Data: These data define the communication channels of the node, i.e., which data should be sent to which of the other nodes in the system. This data are dynamically changed by the meta-agent in run-time, as the situation may require adaptation of the function of the node

Following is a description of network layers included in the communication module (wrapper) part of the node (agent). The corresponding ISO/OSI network layers are shown in Figure 2.

Protocol Y: This protocol defines the communication principles in the system. When it is necessary to send a message to any other node within the system, this protocol defines how the communication should be led.

Mediating Layer: This layer creates an interface between the general part of the communication module and the network-type-dependent part of the module. In a case the module is to be used with a new network type, this and only this part would be changed to meet the specification of the new network type. It means, that all the layers situated above this one should be independent on the network type.

Standard Protocol Stack: This layer is an implementation of a standard stack of the given network protocol.

Network Hardware: A network type dependent hardware equipment. 


\subsection{Reconfiguration}

The problem of system reconfigurability/autoconfigurability is very important in control engineering tasks (Agre, 1995). Unfortunately, there is no unified terminology used in this area. For DC purposes where no automatic active changes in hardware structure are expected the solution of the autoconfiguration task usually consists of two phases:

a) recognition/detection of the event changing current "stable" situation (detection of a node failure or removal, discovery of a new node in the network etc.).

b) automatic reconfiguration of the agents with the aim to optimize the DC performance after the above mentioned event.

One of the significant features of the proposed multi-agent architecture is just its capability of autoconfiguration. The recognition/detection stage can be performed by appropriate meta-agents, e.g. by observing the network/message traffic. There is a wide variety of methods (from simple ad hoc methods to the method of signatures (Chand, 1991) (Chand, 1993)) which can be used to detect the hardware changes in the network or its part.

The reconfiguration phase is aimed exclusively at the software reconfiguration, as all the hardware changes are expected either to be done by a human operator (adding/removing a

1.

2.

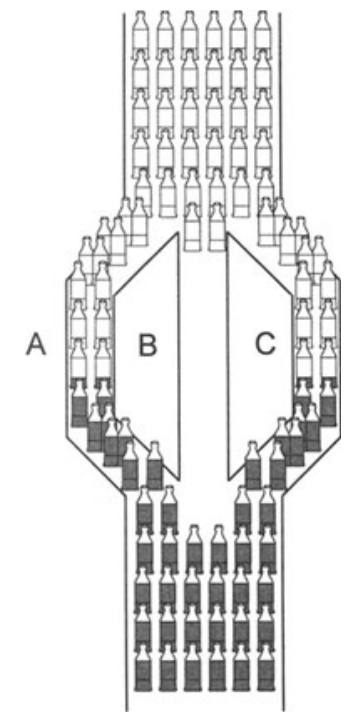

\begin{tabular}{|l|}
\hline 1. conveyor belt transporting empty bottles \\
\hline 2. three filling machines working in parallel \\
\hline 3. conveyor belt transporting full bottles \\
\hline
\end{tabular}

Figure 4.

device) or to occur as a result of hardware failure. The meta-agent is expected to behave in a deliberative style (Wooldridge, 1995): it should make the decision about the new production strategy, then it downloads the necessary new or changed pieces of software configuration data to the corresponding wrappers/devices through the network.

Let's mention here that one meta-agent is usually dedicated to solve autoconfiguration task within a subset of nodes (cluster). 
To demonstrate a typical autoconfiguration task, let's consider a bottling line as shown in Figure 4 . In the case the filling machine B fails due to empty bottle proximity switch failure, the meta-agent responsible for the bottling line performance carries out the following actions:

1. Detection of failure

2. Reconfiguration: The production strategy is changed (all the empty bottles from the conveyor belt transporting empty bottles should be distributed between the filling machines A and $\mathrm{C}$ ), the corresponding changes in the node software are downloaded to the nodes.

3. Detection of a node being repaired: The meta-agent receives the information that the original switch failure was eliminated.

4. Reconfiguration: The original production strategy is accepted again, the necessary pieces of the original software are downloaded back to the corresponding wrappers/devices.

\section{CONCLUSIONS}

This paper gathers the experience of authors who have been involved in both the distributed decision making as well as distributed control engineering research and application projects. The software architecture of the multi-agent decision making system is considered as a guideline for the new architecture of the distributed control systems. Both the AI-based and software engineering oriented viewpoints are expected to help to better understanding of the nature of the multi-agent systems within the community of industrial control engineers. The AI approach stresses namely autonomous behaviour of agents, object-oriented way of communication and demonstrates the opportunity to suppress the role of some central element. Moreover, a good technique for automatic system reconfiguration which is of crucial importance in distributed control architecture is provided by the AI.

The paper demonstrates the opportunity to construct the distributed control systems as nearly an exact analogy to the multi-agent decision making tools. Especially, the role of a wrapper (which contains the model of agent's re-active behaviour and is responsible for communication tasks) is rather stressed and leads to the proposal to dedicate independent hardware elements (processors) for accomplishing the wrapper tasks and device functionalities, respectively. That means that each node (agent) of a distributed control architecture is proposed to consist of two physically separated parts (pluggable modules). As a matter of fact, only very limited number of wrapper types would be needed for industrial tasks. A common control systems producer uses not more than 4 network types and 3 to 4 groups of devices, it would require approximately 20 different wrapper modifications. Considering that it would hardly be attempted to connect some very simple device (a push button) to the highest level network, the number of the wrapper modifications required would be probably kept under 10 . Thus, this solution brings higher modularity to DC systems and reduces the number of necessary component types (lower investments, easier maintenance).

All the behavioral model of a node is contained exclusively in the wrapper - no changes/modifications in the stand-alone devices to be plugged-in into the DC network are needed. Neither central part of memory nor central control algorithm (program) are required. The programs for activity of the agents are downloaded to the wrappers/devices from the workstation which plays - similarly to DISCIM architecture - a role of an independent agent.

The methodology of meta-agents is advantageous for solving the reconfigurability problems. The meta-agents can ensure appropriate software for new agents (by downloading the 
corresponding programs to the newly plugged-in hardware), reconfigure the network or redistribute the load within specific groups (clusters) of agents.

Communication in the case of a distributed control system is highly standardized, but general enough. It supposes introduction of two well-defined languages (language $X$ and proto$\operatorname{col} Y$ ) for the standardized communication between the device and wrapper as well as between the wrapper and network, respectively. This fact supports software reusability.

Besides many features of similarity between the DISCIM environment and the proposed DC architecture there are, of course, some differences visible. Whereas the DISCIM agents explore high-performance multi-purpose computational units, the control subsystems are expected to be much simpler, very often single-purpose devices using much simpler communication languages. That is why, modularity and system integrability seems to be much more important questions in creating the DC systems. Because of the same reason, the problem of system reconfigurability is more crucial in control than in decision making. The other (not very significant) difference is the way of message sending: broadcasting in the case of DISCIM is rather substituted by addressed messaging in the DC architecture. This fact doesn't influence the multi-agent nature of the DC systems, but better reflects the real needs in control.

The higher price of the components (esp. wrappers) is partially compensated by needs of lower maintenance costs and smaller spare parts stocks. Moreover, the prices (especially of the processors) are dropping down permanently and dramatically. It is no problem to pay higher price for the development environment if the probability of price drop in the future is high enough. The advanced users are already asking for highly flexible control solutions and they are even willing to pay more for such solutions, because they feel that the use of these new technologies might bring them a competitive advantage. Also, there are currently devices on the market that are very suitable for the newly proposed control architecture. This fact should simplify the transfer of the above mentioned ideas to the industrial environment.

\section{REFERENCES}

Afsarmanesh H., Wiedijk M., Hertzberger L. (1994) Flexible and Dynamic Integration of Multiple Information Bases. In: Proc. of the 5th International DEXA'94 Conference, LNCS No. 856, Springer-Verlag, Heidelberg, 744-753

Agre J., Clare L., Lee J., Brandin B., Hoskins J., Perrone M. (1995) Autoconfigurable Distributed Control Systems. In: Proc. of 2nd Int. Symposium on Autonomous Decentralized Systems (ISADS'95), Phoenix, AZ, 162-168

Chand S. (1991) Observers for Discrete-Event Manufacturing Systems. In: Proc. of the IECON 91 Conference. Kobe

Chand S. (1993) Discrete-Event Based Monitoring and Diagnosis of Manufacturing Processes. In: Proc. of the 1993 American Control Conference, San Francisco, CA

de Kleer J., Weld D.S. (1990) Readings in Qualitative Reasoning about Physical Systems, Morgan Kaufmann Publishers, Inc., San Mateo, CA

(ECL $\left.{ }^{i} \mathrm{PS}{ }^{e}, 1992\right):$ Eclipse 3.3 User Manual (ECRC Common Logic Programming System), ECRC, Munich

Hazdra T., Koutník J. (1995) Graphical Interface for Distributed Expert System, Technical Report OAKBS-95/12, FAW J. Kepler University, Linz 
Holas D., Mařík V. (1995) Evaluation of the First Experiments with the fm/Aspects Tool. In: Proceedings of the 6th International DEXA'95 Conference, Omnipress Publishing, Madison, WI, 439-447

Jennings N.R., Witting T. (1993) ARCHON: Theory and Practice. In: Distributed AI: Theory and Praxis (eds. Avouris M., Gasser L.), Kluver Academic Publishers, Dordrecht, 179195

Mařik V. and others (1988) Application of the FEL-EXPERT System in the Diagnosis of Genetic Diseases. In: Advances in Biomedical Measurement (eds. Carson R.E., Kneppo P., Krekule I.), Plenum Press, New York, 465-475

Mařík V., Lažanský J., Koutník J., Hazdra T. (1994) Distributed Systems for CIM. In: Proc. of 5th International Conference DEXA'94, LNCS No. 856, Springer-Verlag, Heidelberg, 785-794

Maturana F.P., Norrie D.H. (1996) Multi-Agent Coordination Using Virtual Clustering in a Distributed Manufacturing System. In: Proceedings of 5th Industrial Engineering Research Conference, Minneapolis, $\mathrm{MN}$

O'Hare, G.M.P., Wooldridge, M.J. (1993) A Software Engineering Perspective on MultiAgent System Design. In: Distributed AI: Theory and Praxis (eds. Avouris M., Gasser L.), Kluver Academic Publishers, Dordrecht, 109-128

Osorio A.L., Camarinha-Matos L.M. (1995) Support for concurrent engineering in CIM-FACE. In: Balanced Automation Systems (eds. Camarinha-Matos L.M. and Afsarmanesh H.), Chapman \& Hall, London, 275-286

(PVM, 1993): A User's Guide To PVM. Oak Ridge National Laboratory

Rabelo R.J., Camarinha-Matos L.M. (1995) HOLOS: A Methodology for Deriving Scheduling Systems. In: Balanced Automation Systems (eds. Camarinha-Matos L.M. and Afsarmanesh H.), Chapman \& Hall, London, 181-194

Rao M. (1992) Integrated Systems for Intelligent Control. Springer-Verlag, Berlin

Štěpánková O., Marvan I. (1994) Time Independent Global Constraints and QSIM.In: Proc. of ESM Conf., Barcelona, 1994, 470-474

Štěpánková O. (1995) Model Identification through Qualitative Reasoning: A Case Study. In: Proc. of Artificial Intelligence Techniques AiT'95 (eds. Žižka J. and Brazdil P.), TU Brno, 103-112

Wooldridge M.J. and Jennings N.R. (1995) Agent Theories, Architectures, and Languages: A Survey. In: Intelligent Agents (eds. Wooldridge M.J. and Jennings N.R.), LNAI No. 890, Springer-Verlag, Heidelberg, 1-39 